\title{
Hepatic Infarction Caused by Antiphospholipid Syndrome Secondary to Systemic Lupus Erythematosus
}

CAN LI ${ }^{\circ}$, MD; JIULIANG ZHAO, MD; YAN ZHAO, MD, Department of Rheumatology, Peking Union Medical College Hospital, Beijing, China. Address correspondence to Dr. J. Zhao, Department of Rheumatology, Peking Union Medical College Hospital, No. 1 Shuaifuyuan, Dongcheng District, Beijing 100730, China. E-mail: zjlpumc@ sina.com. This work was approved by the Ethics Committee of Peking Union Medical College Hospital (S-191), and written consent from the patient was obtained for publication. The work was supported by The Peking Union Medical College Hospital Fund for Distinguished Young Scholars (JQ201706). J Rheumatol 2019;46:755-6; doi:10.3899/jrheum.181241

Hepatic infarction is a rare complication of systemic lupus erythematosus (SLE) ${ }^{1}$.

A 29-year-old man diagnosed with SLE was admitted to our hospital with abdominal pain and fever. A computed tomography scan indicated multiple round hypodense lesions in the liver (Figure 1A). We suspected liver abscess. Broad-spectrum antibiotics were applied for 2 weeks but were ineffective.
Liver function results showed total bilirubin was 26.1 $\mu \mathrm{mol} / \mathrm{l}$, gamma-glutamyl transpeptidase was $211 \mathrm{U} / 1$, and glutamic-pyruvic transaminase and aspartate transaminase were normal. Magnetic resonance imaging (MRI) showed multiple hypointense lesions along hepatic vessels on T1-weighted (T1W) image (Figure 1B). Liver biopsy revealed confluent necrosis and microthrombi in arterioles (Figure 2A). Additionally, hemosiderin was identified with

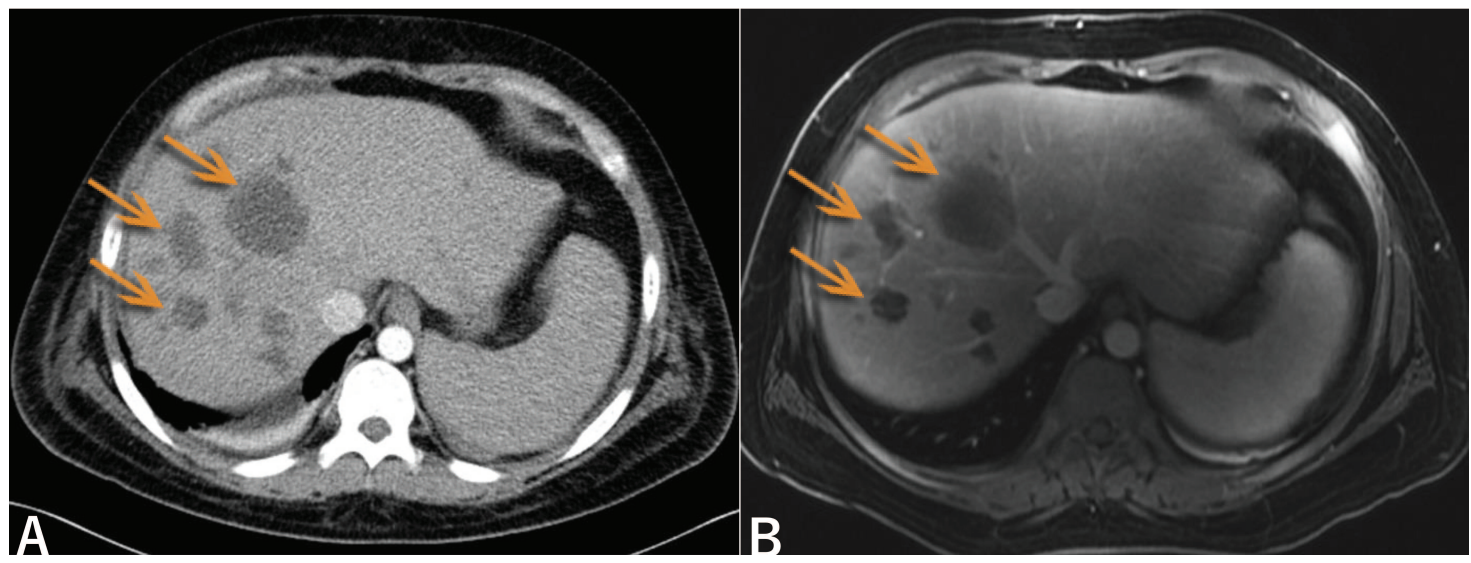

Figure 1. Image test results of hepatic infarction caused by systemic lupus erythematosus. A. A CT scan indicated multiple round hypodense lesions (arrows) in the liver. B. At the same level, MRI showed multiple hypointense lesions distributed along hepatic blood vessels (arrows) on T1-weighted image. CT: computed tomography; MRI: magnetic resonance imaging.

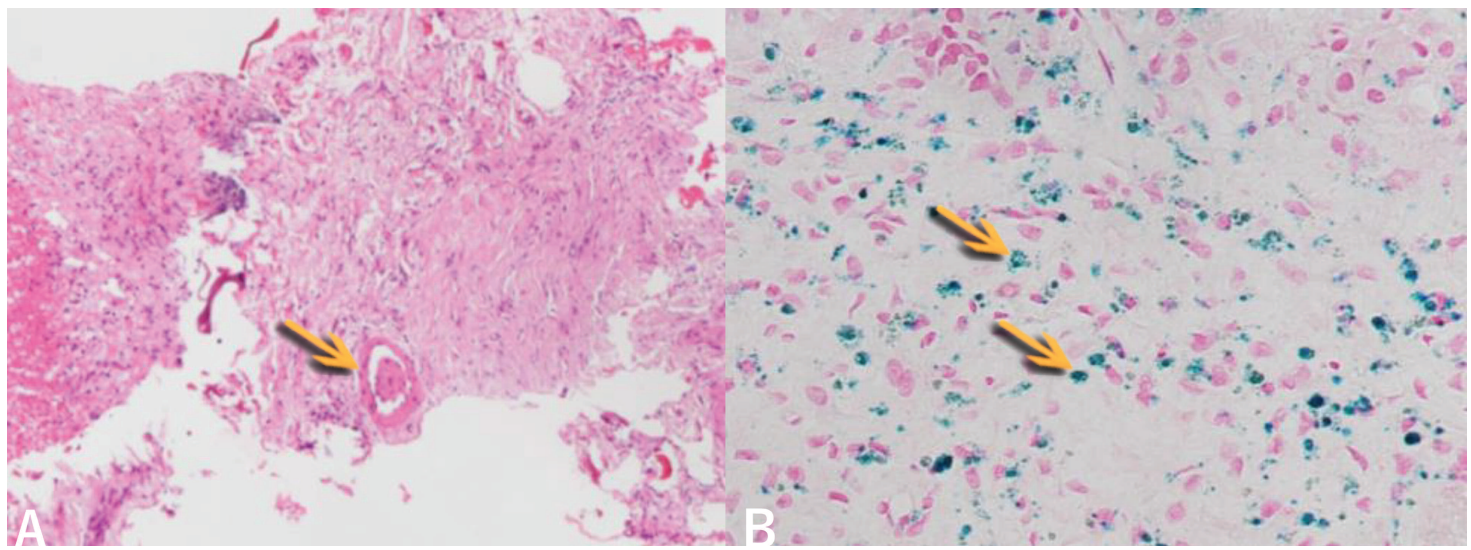

Figure 2. The liver biopsy result of hepatic infarction caused by systemic lupus erythematosus. The liver biopsy revealed necrosis, and (A) microthrombus in the arteriole (arrow), and (B) hemosiderin was identified with Perls' staining (arrows). 
Perls staining (Figure 2B). Lupus anticoagulant was 2.45 (normal range: $\leq 1.2$ ) and continually positive, whereas anticardiolipin antibodies and anti- $\beta_{2}$-glycoprotein I antibodies were negative.

The patient was diagnosed with antiphospholipid syndrome (APS) secondary to SLE, manifesting with hepatic avascular necrosis. He was prescribed glucocorticoid impulsive therapy, mycophenolate mofetil, intravenous immunoglobulin, and anticoagulation treatment, all of which led to clinical improvement. The lesions had been considerably reduced as shown on the repeated MRI 3 months later.

Patients with SLE may develop vasculitis ${ }^{2}$. Our patient developed secondary APS, thereby elevating the risk of thrombosis ${ }^{3}$. These 2 reasons contributed to the development of a hepatic infarction. Hepatic infarction could be misdiag- nosed as liver abscesses because both conditions present as hypointense lesions on T1W images. Different from the mass effect of liver abscesses, however, lesions of hepatic infarction develop along the biliary duct, portal vein, and hepatic artery, with vessels passing through.

\section{REFERENCES}

1. Matsumoto T, Yoshimine T, Shimouchi K, Shiotu H, Kuwabara N, Fukuda Y, et al. The liver in systemic lupus erythematosus: pathologic analysis of 52 cases and review of Japanese Autopsy Registry Data. Hum Pathol 1992;23:1151-8.

2. Ebert EC, Hagspiel KD. Gastrointestinal and hepatic manifestations of systemic lupus erythematosus. J Clin Gastroenterol 2011; 45:436-41

3. D'Cruz DP, Khamashta MA, Hughes GR. Systemic lupus erythematosus. Lancet 2007;369:587-96. 